\title{
Modelo para la ordenación paisajística del espacio periurbano
}

\section{Methodological model for landscape planning in peri-urban areas}

\author{
M. Gómez Villarino ${ }^{(*)}$, T. Gómez Villarino ${ }^{(*)}$
}

\section{RESUMEN}

Se presenta un modelo metodológico para analizar, interpretar y planificar el paisaje del espacio periurbano, fruto de la investigación aplicada que vienen desarrollando los autores sobre diversos espacios; aquí se describe el último de ellos.

Su interés estriba en la importancia creciente del periurbano, y en el déficit metodológico del estudio de su paisaje, en contraste con la abundancia en territorios supraurbanos.

El modelo estructura de forma coherente y comprensiva, conceptos paisajísticos diferentes, en tres niveles y un doble carácter: conceptual y metodológico; conceptualmente los niveles corresponden a tres grados de aproximación al conocimiento del paisaje: descriptivo, interpretativo y propositivo; y metodológicamente, a las tres fases de formulación de un plan: análisis, diagnóstico y propuestas. Además considera las facetas rural y urbana conformadoras del periurbano y las dos áreas de atención que requiere el paisaje: la base paisajística y la percepción.

Palabras clave: Periurbano, Paisaje, Urbano, Rural, Modelo, Metodología.

\section{ABSTRACT}

A methodology is presented to analyze, diagnose and plan the urban landscape, as a result of the applied research that the authors are developing on diverse spaces; the one that is described along the text, is the last one.

Its interest relies on the increasing importance of the periurban and in the methodological deficit of the study of its landscape, in contrast to the abundance in supra-urban territories.

The model structures coherently and comprehensively, different landscape concepts, in three levels and double character: conceptual and methodological; Conceptually the levels correspond to three degrees of approximation to the knowledge of the landscape: descriptive, interpretative and propositive; And methodologically, to the three phases of formulating a plan: analysis, diagnosis and proposals. In addition it considers the rural and urban facets that make up the periurban and the two areas of attention that the landscape requires: the landscape base and the perception.

Keywords: Peri-urban, Landscape, Urban, Rural, Model, Methodology.

(*) Universidad Politécnica de Madrid.

Persona de contacto/Corresponding author: teresa.gomez.villarino@upm.es (T. Gómez Villarino)

ORCID: http://orcid.org/oooo-0oo2-2887-6077 (M. Gómez Villarino); http://orcid.org/oooo-0002-8720-3593

(T. Gómez Villarino)

Cómo citar este artículo/Citation: M. Gómez Villarino; T. Gómez Villarino. (2018). Modelo para la ordenación paisajística del espacio periurbano. Informes de la Construcción, 70(550): e250. https://doi.org/10.3989/ic.16.081

Copyright: ( $\mathbf{2 0 1 8}$ CSIC. Este es un artículo de acceso abierto distribuido bajo los términos de la licencia de uso y distribución Creative Commons Reconocimiento 4.o Internacional (CC BY 4.0). 


\section{INTRODUCCIÓN Y OBJETIVOS}

La experiencia de los autores adquirida a través de numerosos trabajos profesionales sobre paisaje en espacios supraurbanos, metropolitanos y urbanos, incluyendo núcleos rurales, ha puesto de manifiesto la confluencia de intereses conflictivos sobre el espacio periurbano, su consiguiente tendencia a la degradación ambiental, territorial y paisajística, y su repercusión en la imagen que transmite de la ciudad a quien se aproxima a ella.

Esta percepción empírica del periurbano se corrobora por el creciente interés social que suscita y por su reflejo en la bibliografía al respecto; el Observatorio Regional de los Paisajes, Región Rhóne-Alpes, Francia (1), incluye los periurbanos entre las siete familias de paisajes paradigmáticos; Tveit y Ode (2) señalan la relevancia del periurbano en ámbitos metropolitanos, y hacen referencia a otros autores que se manifiestan en el mismo sentido (3) (4); y diversas escuelas de paisajismo y planeamiento urbanístico focalizan su atención hacia ámbitos aledaños al urbano (5) (6) (7) (8).

El periurbano es un espacio frontero entre la ciudad y su entorno rural, de límites imprecisos, pero con funciones y actividades peculiares que le dotan de entidad propia, y que reclama planificación y gestión específicas. Se trata de un sistema territorial, y como tal, "construido" por el hombre a través de las actividades que se practican sobre él, cual son: las que la ciudad expulsa, por incompatibilidad, precio del suelo, rechazo social, etc.; las que aprovechan la proximidad de una demanda urbana: centros hípicos, caninos, felinos, recreo al aire libre, etc.; actividades rurales que permanecen; y actividades indeseables que aprovechan el vacío de regulación para ubicarse en él.

Sin embargo, su límite es difuso; el interno, aun sometido a las múltiples formas de expansión urbana, queda administrativamente delimitado por el planeamiento urbanístico; pero el externo exige definir hasta dónde llega el carácter periurbano de las actividades específicas. Esta indefinición aconseja delimitar el periurbano en función de los objetivos que se pretendan en cada caso, y recurrir al consenso entre los implicados considerando dos criterios: la frecuencia e inmediatez de las relaciones con el urbano y la vinculación directa a él por la distancia.

En cuanto al paisaje, desde que el Consejo de Europa aprobó el Convenio Europeo del Paisaje (9) (10), y que otras regiones siguieran su estela, como la Iniciativa Latinoamericana del Paisaje (11), ha pasado de tema menor a dimensión sustantiva del sistema territorial, a indicador de salud ambiental y de prestigio social, a recurso socioeconómico y a factor de localización de actividades económicas de vanguardia.

Todo ello ha suscitado el interés por estudiar el paisaje y gestionar su formación a futuro, poniendo de manifiesto dos grandes dificultades: la condición polisémica del término paisaje y su carácter de percepción subjetiva (12) (13). Estas dificultades aconsejan fijar el concepto de paisaje que aplica este texto, recurriendo para ello al Convenio Europeo del Paisaje, que lo entiende así "cualquier parte del territorio, tal como lo perciben las poblaciones". Más intencionadamente se puede expresar como la percepción polisensorial y subjetiva del sistema territorial, expresión que implica que el paisaje, como el sistema territorial, es una construcción humana inexorable. Esta idea de paisaje, sugiere dos esferas de estudio: una objetiva, la imagen que trasmite el sistema territorial, a la que denominamos base paisajística; otra subjetiva: la percepción de ella, fundamentalmente visual, por los observadores potenciales.

Existe abundante bibliografía sobre metodología y técnica para territorios supraurbanos; por ejemplo, el ya clásico "landscape carachter assesment", en Reino Unido, los Atlas de Paisaje en Francia (14) o las iniciativas de diversas comunidades autónomas españolas (15). Más escasa es la bibliografía referida al paisaje urbano; y aún más al periurbano; y la que hay sobre éste, generalmente parcial: orientada a definir "zonas de protección de vistas" -Plan de Paisaje de Segovia(16), a establecer corredores paisajísticos -Plan Intercomunal de Temuco, Chile- (17), a mejorar el paisaje en travesías y accesos a pueblos (18); o se refieren a ámbitos metropolitanos aunque incluyan espacios periurbanos (2) (3) (4), o a la recuperación paisajística de espacios residuales (19) (20) (21); otros autores incursionan específicamente en el periurbano, con escaso reflejo de la interconexión rural-urbano, o focalizando hacia la valoración, frecuentemente estética, o avanzan en el diagnóstico hasta la fragilidad visual o paisajística (22) (23), pero raramente se alcanza la fase de formulación de propuestas para construir el paisaje futuro.

A rellenar las lagunas señaladas pretende contribuir el modelo que se presenta; se concreta (figura 1) en un diagrama de flujos que define un "sistema coherente de tareas" (24) a través de las cuales se va elaborando el plan. Su desarrollo ha permitido confirmar las hipótesis iniciales de trabajo, que apuntaban la posibilidad de expresar el carácter subjetivo del paisaje en conceptos lógicos fácilmente comprensibles, de traducirlo a criterios suficientemente objetivables para inventariarlo, diagnosticarlo y planificarlo, y de ordenarlos en una secuencia lógica de tareas concatenadas que conforman una metodología operativa de planificación paisajística.

\section{MÉTODO DE TRABAJO Y CASO DE APLICACIÓN}

El modelo se ha desarrollado mediante el método inductivodeductivo, observando la realidad, a través de numerosos trabajos profesionales, para conceptualizar lo observado y traducirlo a procesos operativos aplicables a cualquier zona; así su aplicación iterativa va perfeccionando el modelo. Es el método denominado "investigación-acción", donde teoría y práctica se refuerzan mutuamente, desarrollado por Lewin (25) desde 1946. Y se ha entrelazado con un intenso y extenso trabajo de participación social realizada telemáticamente, en talleres formales de participación y en consulta a un panel de expertos multidisciplinar.

Como se ha señalado, el modelo se ha ido elaborando a lo largo de varios años en un proceso de aprendizaje continuo sobre diversos casos reales; el que se presenta en este artículo, es el último de ellos, que ha llevado a su versión actual; se trata de la aplicación a un pueblo rural, Monreal de Ariza, Zaragoza (España), relativamente pequeño, 62,1 Km2 y unas 500 viviendas y edificios, que cuenta con un periurbano (figura 2) reducido pero complejo y bien definido, que manifiesta con claridad la tendencia a la degradación paisajística en numerosos núcleos rurales, donde coexisten un casco interior y un entorno rural bien conservados separados por una franja de expansión urbana difusa, paisajísticamente banal.

La información para elaborarlo procede, fundamentalmente, de trabajo de campo, de fotografía aérea de diferentes fechas, 


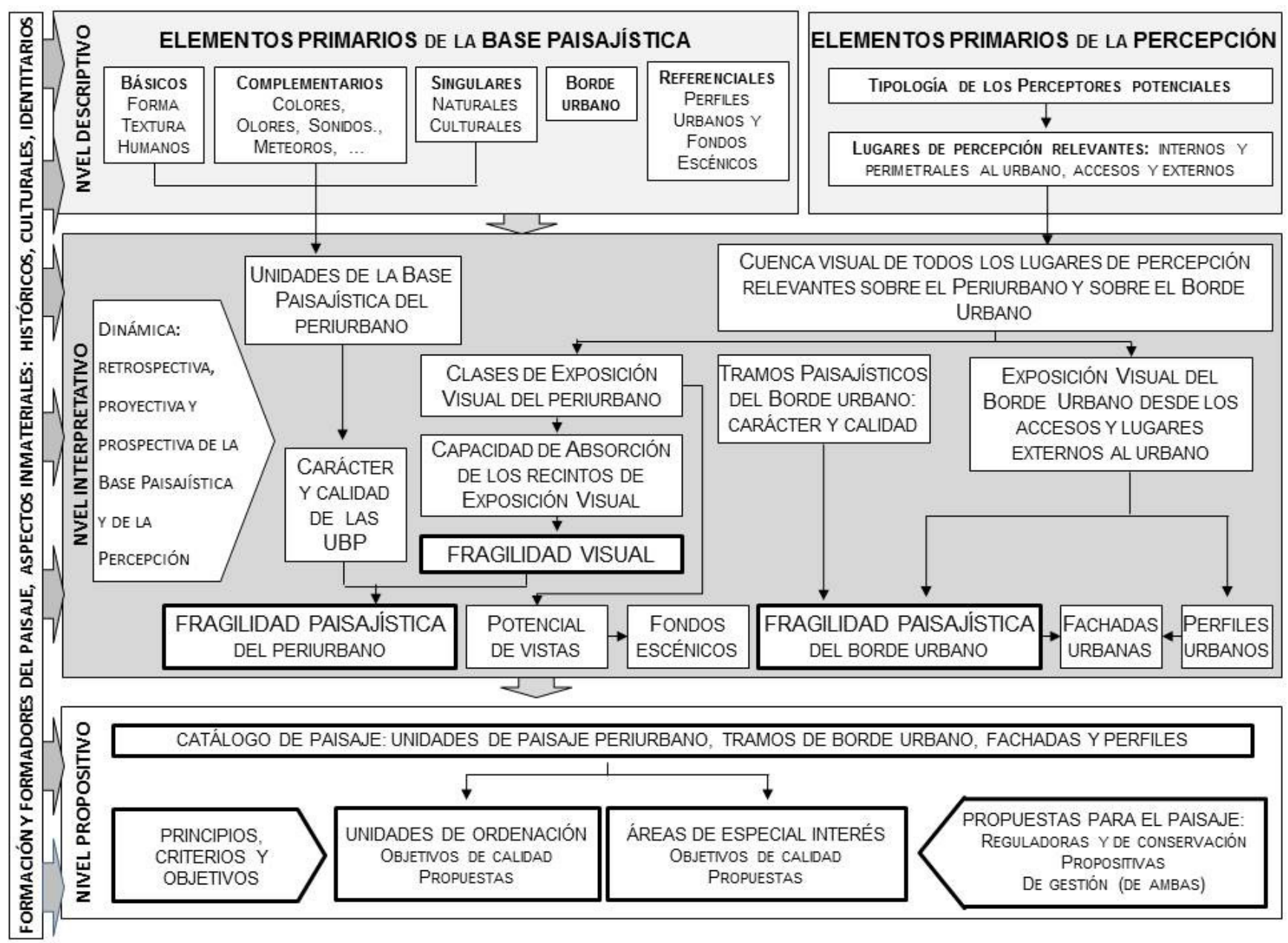

Figura 1. Modelo sintético (conceptual y metodológico) para la ordenación paisajística del espacio periurbano de pueblos y ciudades. Fuente: Elaboración propia.

de modelos digitales del terreno disponibles en la Cobertura LIDAR del Plan Nacional de Ortofotografia Aérea y de la información gráfica que aportan herramientas de uso público como Google Earth o Google Street View; además se han consultado las fuentes que se citan en la bibliografía: (26) (27) (28) (29) (30) (31) y (32). Y se ha aplicado a través de una herramienta GIS.

\section{RESULTADOS: PRESENTACIÓN DEL MODELO Y SU APLICACIÓN AL CASO DE ESTUDIO}

Se describe aquí la última versión del modelo (validada en los entornos académicos, científico y profesional de los autores, así como por la población local), que se va ilustrando con la exposición sintética de la aplicación al caso que sigue a cada epígrafe.

El modelo incluye una doble dualidad: la consideración conjunta de lo urbano y lo rural, y las dos esferas atención que requiere el: la base paisajística y su percepción; se sintetiza en un solo diagrama, a su vez, con un doble carácter (figura 1): uno conceptual que define los elementos que conforman el paisaje periurbano; otro metodológico, que define la secuencia lógica de para formular planes de ordenación paisajístico. Estructuralmente, el modelo se organiza en tres niveles de aproximación, de un lado, al conocimiento del paisaje: descriptivo, interpretativo y propositivo; de otro, a las tres fases de formulación de un plan: información, diagnóstico y propuestas. Una tira vertical, en la izquierda del diagrama, recoge los aspectos históricos, culturales e identitarios, que informa a todos los niveles, conceptos y tareas.

La aplicación del modelo hace uso continuo de la técnica de superposición (con una herramienta GIS), que facilita la comprensión del proceso y de sus resultados por cualquier interesado, pero induce a una superposición mecánica de capas cartográfica, que se evita mediante una aplicación iterativa, avanzando y retrocediendo para revisar lo realizado a la luz del sentido común y de la participación social.

\section{NIVEL DESCRIPTIVO DEL MODELO}

Incluye los elementos básicos de la base paisajística y de la percepción, los cuales se cartografían, caracterizan y valoran en esta fase.

\subsection{Elementos primarios de la Base Paisajística del periurbano}

Se manifiestan de forma directa y permanente al observador. Son estos:

Elementos básicos: aquellos de presencia extensa y permanente, concretamente:

- Formas del terreno, resultado de procesos naturales y antrópicos sobre la corteza terrestre. 


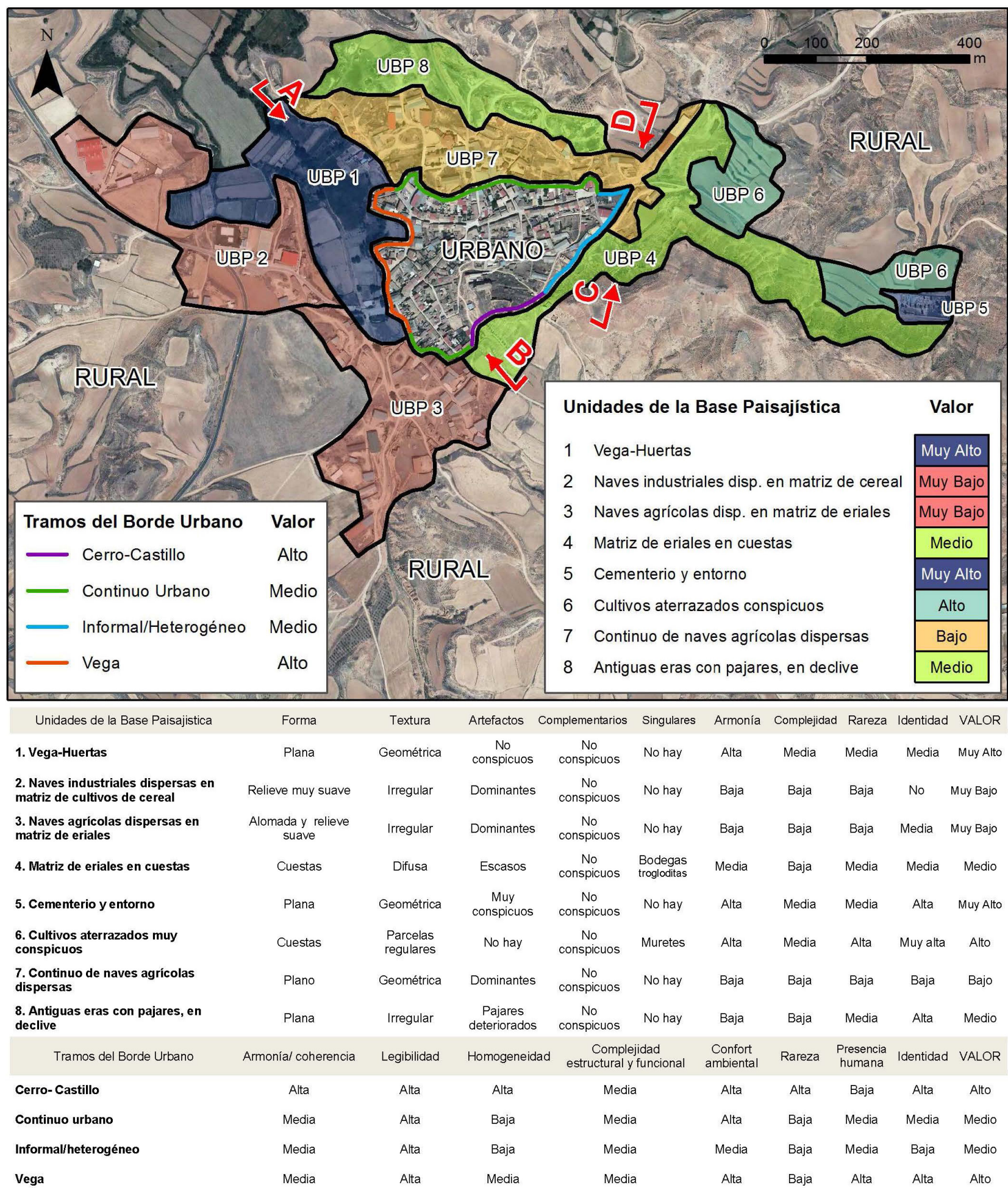

Figura 2. Delimitación y valoración de las Unidades de la Base Paisajística (UBP) del espacio periurbano y de los Tramos Paisajísticos del borde urbano. Además se incluye la situación de los cortes A-B y C-D cuyos respectivos perfiles muestra la figura 4. Fuente: Elaboración propia.

- Textura de la superficie: disposición de los elementos en la superficie de edificios y terrenos.

- "Artefactos" (33) construidos por el hombre: edificios, infraestructuras, equipos, etc.

Elementos complementarios: menos significativos que los básicos, y temporales o esporádicos:

- Colores, dominancia, combinación armónica o discordante de colores.

- Olores, gratos, desagradables, continuos, cíclicos, etc.
- Sonidos/ruidos, que pueden dar identidad a ciertas zonas, como las próximas a accesos.

- Meteoros, o la propia luz: nieblas, transparencia, etc.

- Percepciones telúricas: atmósfera emocional en ciertos ámbitos culturales, atávicos, etc.

- Presencias visuales esporádicas: animales silvestres (como paso de aves migratorias) o domésticos, etc.

Elementos singulares: de escasa entidad superficial pero fuerte relevancia visual, cultural, histórica o identitaria. 
Borde urbano: línea perimetral del urbano más o menos consolidado, definida, administrativamente, por la clasificación urbanística del suelo; desde el terreno se manifiesta en un alzado.

Referenciales, constituyen referencias muy estables que orientan al observador. Se consideran dos:

- Perfil urbano: línea de horizonte delimitada por el ritmo altitudinal y horizontal de los edificios, que se percibe desde los accesos más importantes.

- Fondos Escénicos: barreras visuales permanentes que cierran el horizonte lejano.

\section{APLICACIÓN AL CASO DE ESTUDIO}

Tres formas presenta el terreno estudiado: plana de vega, suavemente ondulada y cuestas alteradas por la erosión; la textura es geométrica en las parcelas de cultivo en la vega y e informal en el resto; los artefactos son naves agrícolas, industriales o logísticas informalmente ubicadas; destaca el cementerio municipal, conectado al pueblo por un camino muy frecuentado. No existen elementos complementarios ni singulares relevantes.

El borde urbano presenta los tramos señalados en la figura 2.

La línea roja de la figura 3 marca dos perfiles: arriba, el característico (histórico) del pueblo; abajo otro, más banal, representa la evolución actual; la línea negra marca el perfil del terreno.

La figura 4 muestra la visual sobre el fondo escénico referencial que conforma la sierra.

\subsection{Elementos primarios de la Percepción}

El modelo considera los perceptores potenciales del paisaje y los lugares relevantes para la percepción.
Identificación y caracterización de los perceptores potenciales, mediante observación y participación ciudadana; genéricamente se agrupan en:

- Residentes: "formadores" del paisaje, y usuarios habituales.

- Foráneos: visitantes ocasionales, turistas, emigrados, etc.

- Expertos, planificadores y gestores del paisaje.

- Particulares. implicados en actividades económicas relacionadas con el paisaje.

- Actores sociales o gestores de la ciudadanía, de carácter cultural, social, político, etc.

Identificación y cartografía de los lugares relevantes para la percepción y caracterización por el tipo y cantidad de perceptores potenciales: puntos, rutas y áreas internos, perimetrales o externos al urbano:

- Accesos por carretera o ferrocarril, salidas desde estaciones, aeropuertos ...

- Miradores hacia el exterior y hacia los fondos escénicos.

- Perímetro urbano.

- Viario rural en el periurbano.

- Itinerarios frecuentados a pie, en bicicleta, a caballo, etc.

\section{APLICACIÓN AL CASO DE ESTUDIO}

Los perceptores potenciales del paisaje en este caso pertenecen a todas las categorías señaladas; destacan los emigrados que vuelven al pueblo en fechas señaladas o vacacionales.

Como lugares de percepción relevantes se han identificado y cartografiado (figura 4), los miradores del castillo y la antigua iglesia, los caminos del cementerio y de la vega y los accesos al pueblo.

\section{NIVEL INTERPRETATIVO DEL MODELO}

Los conceptos incluidos aquí se refieren, como siempre, a la base paisajística, y a la percepción.
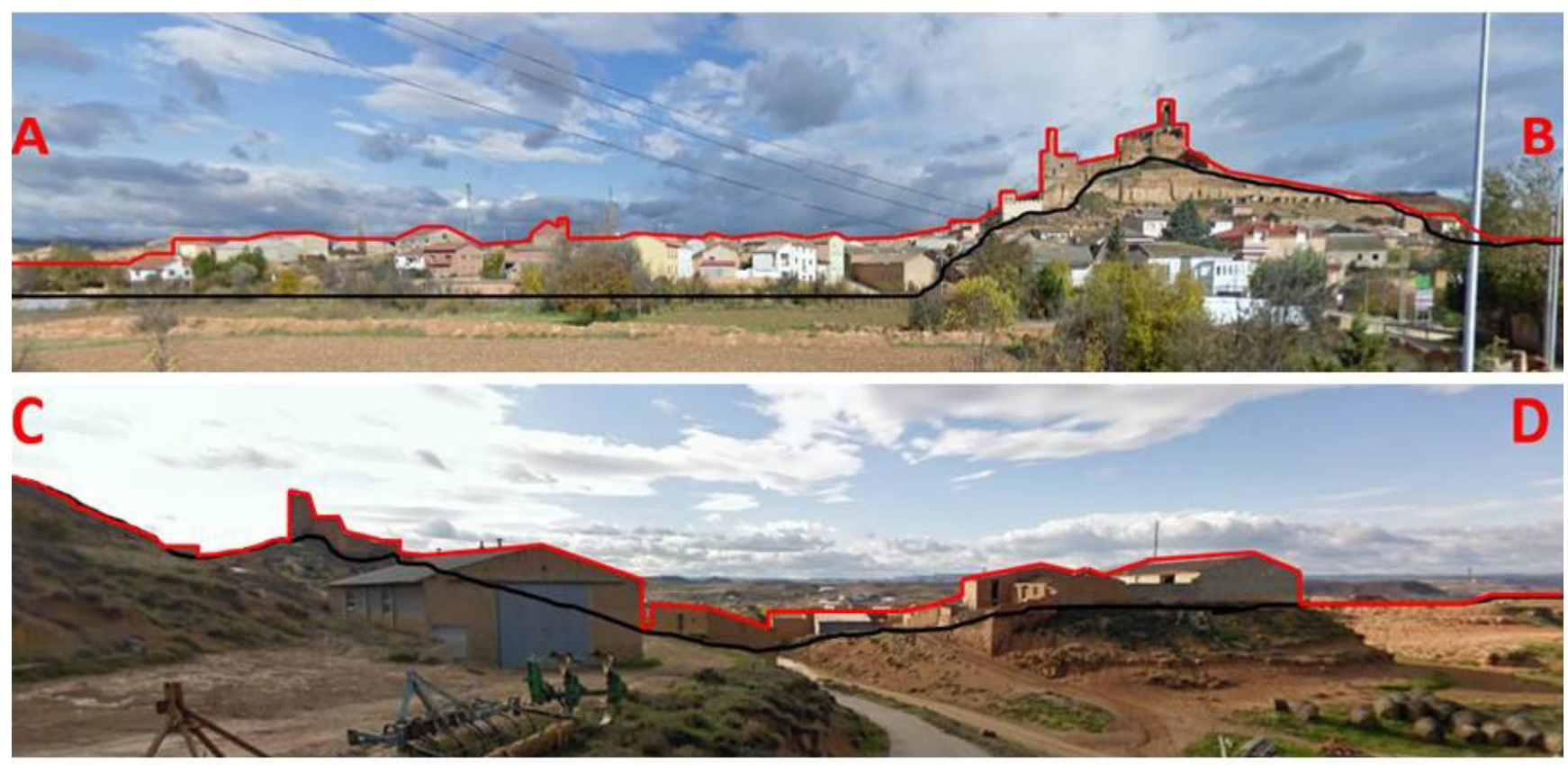

Figura 3. Perfiles urbanos correspondientes a los cortes A-B y C-D referenciados en la figura 2; en negro perfil del terreno, en rojo perfil de la edificación. Fuente: Elaboración propia. 


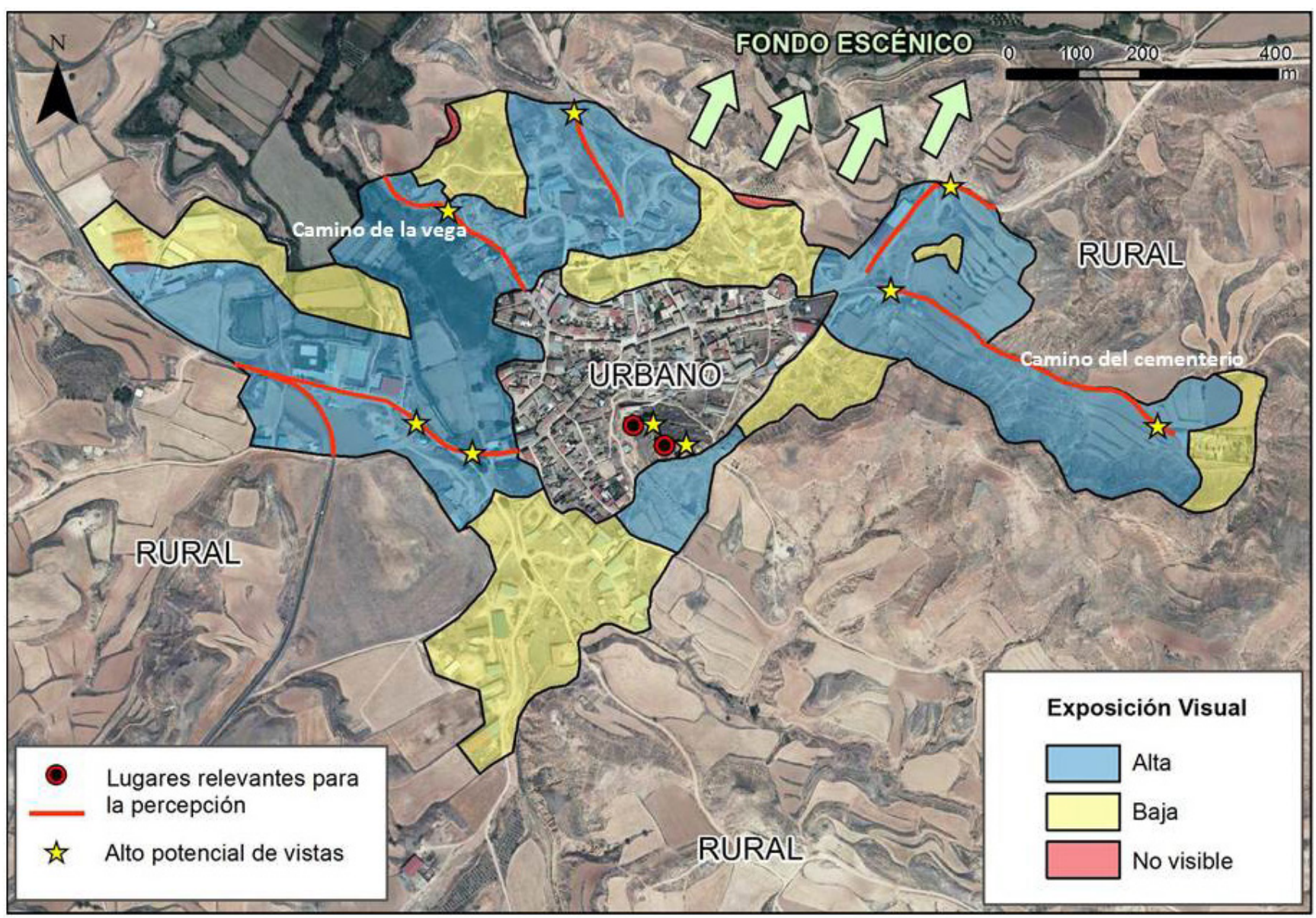

Figura 4. Mapa representando los Lugares Relevantes para la Percepción, las clases y recintos de Exposición Visual del periurbano desde ellos, los Puntos con Alto Potencial de Vistas y las visuales hacia el Fondo Escénico referencial. Fuente: Elaboración propia.

\subsection{Elementos interpretativos de la Base Paisajística}

Unidades de la Base Paisajística (UBP) del periurbano. Son porciones del periurbano definidas por su "carácter", es decir, por aquello que la diferencia del resto. Se identifican a partir de la yuxtaposición de los rasgos descriptivos, identificados en la fase anterior, comenzando por los más significativos: formas, textura y aspectos histórico-culturales.

Carácter y calidad de las UBP. El carácter de una unidad viene definido por los rasgos que permitieron identificarla; su calidad se refiere a sus "méritos de conservación": aquello que hace que deba ser conservada, deba evolucionar conservando su carácter o pueda cambiarlo sin merma de valor; o con mejora. La bibliografía consultada (34) (35) (36) (37), y nuestra experiencia extraída de participación ciudadana, sugieren los siguientes indicadores de valoración, que deberán ajustarse en cada caso: armonía, coherencia, historicidad, complejidad, homogeneidad, legibilidad, naturalidad, confort ambiental, discordancias, rareza, seguridad, efímeros y elementos de percepción no visuales.

Dos enfoques complementarios se vienen utilizando para valorar el paisaje (38) (39) (40): uno técnico, basado en la desagregación en componentes; otro participativo mediante consulta directa a los observadores potenciales sobre la calidad de cada unidad.

Tramos Paisajísticos del Borde urbano: carácter y calidad. La heterogeneidad del borde urbano sugiere identificar tra- mos paisajísticos de borde, equivalentes a unidades lineales de paisaje, y valorarlos mediante los indicadores señalados.

\section{APLICACIÓN AL CASO DE ESTUDIO}

La figura 2 muestra la localización, caracterización y valoración de las UBP del periurbano y de los Tramos paisajísticos del Borde urbano; la valoración combinó los enfoques técnico y participativo.

\subsection{Elementos interpretativos de la Percepción}

Las relaciones intervisuales entre los espacios urbano y periurbano se interpretan a través de los aspectos siguientes:

Cuenca visual de todos los lugares de percepción relevantes sobre el periurbano. Cuenca visual es el terreno que se ve desde un punto, itinerario o área; se determina a partir del modelo digital del terreno en función del relieve, la distancia y las barreras visuales.

Exposición visual del periurbano. Se refiere a la capacidad "emisora" de vistas o "potencial de ser visto", del terreno; se representa dividiendo las cuencas visuales en clases de mayor a menor visibilidad, originando numerosos recintos de exposición visual

Capacidad de Absorción de los recintos de exposición visual. Es la capacidad del terreno para incorporar elementos añadidos sin detrimento de su calidad. Se identifica, cartografía 
y valora en función de la densidad y envergadura de la cobertura vegetal y de la complejidad del microrelieve, superponiendo las capas cartográficas correspondientes a ambos aspectos y aplicando los cruces que muestra la figura 5. Éstos cruces manifiestan la siguiente lógica: una zona arbolada con ejemplares de gran tamaño "absorbe" con facilidad elementos extraños de moderado volumen; y lo mismo un microrelieve complejo, compartimentado.

Exposición Visual del Borde Urbano desde los accesos y lugares externos al urbano. Aplicación del concepto de exposición visual al borde urbano desde accesos y lugares externos al urbano.

\section{APLICACIÓN AL CASO DE ESTUDIO}

La figura 4 muestra las clases de exposición visual del periurbano desde los lugares relevantes para la percepción, identificadas a partir de sus cuencas visuales, y los recintos de exposición visual a que da lugar su representación cartográfica.
El mismo análisis de exposición visual se aplica al borde urbano, pero ahora solamente desde los lugares de percepción externos: accesos y caminos de la vega y del cementerio. El perímetro de contacto de los recintos de exposición visual, en la propia figura 4, corresponde a la exposición visual del borde urbano, y por tanto de sus tramos, representados en la figura 2.

La figura 5 muestra el mapa de capacidad de absorción obtenido por superposición de las capas cartográficas correspondiente al microrelieve y a la cobertura vegetal, y aplicando los cruces de la tabla.

\subsection{Elementos interpretativos que combinan la Base Paisajística y la Percepción}

Situados al final del nivel interpretativo, estos elementos suponen tal detalle de elaboración que permiten acceder fácilmente a las decisiones en el nivel propositivo. Se describen a continuación:
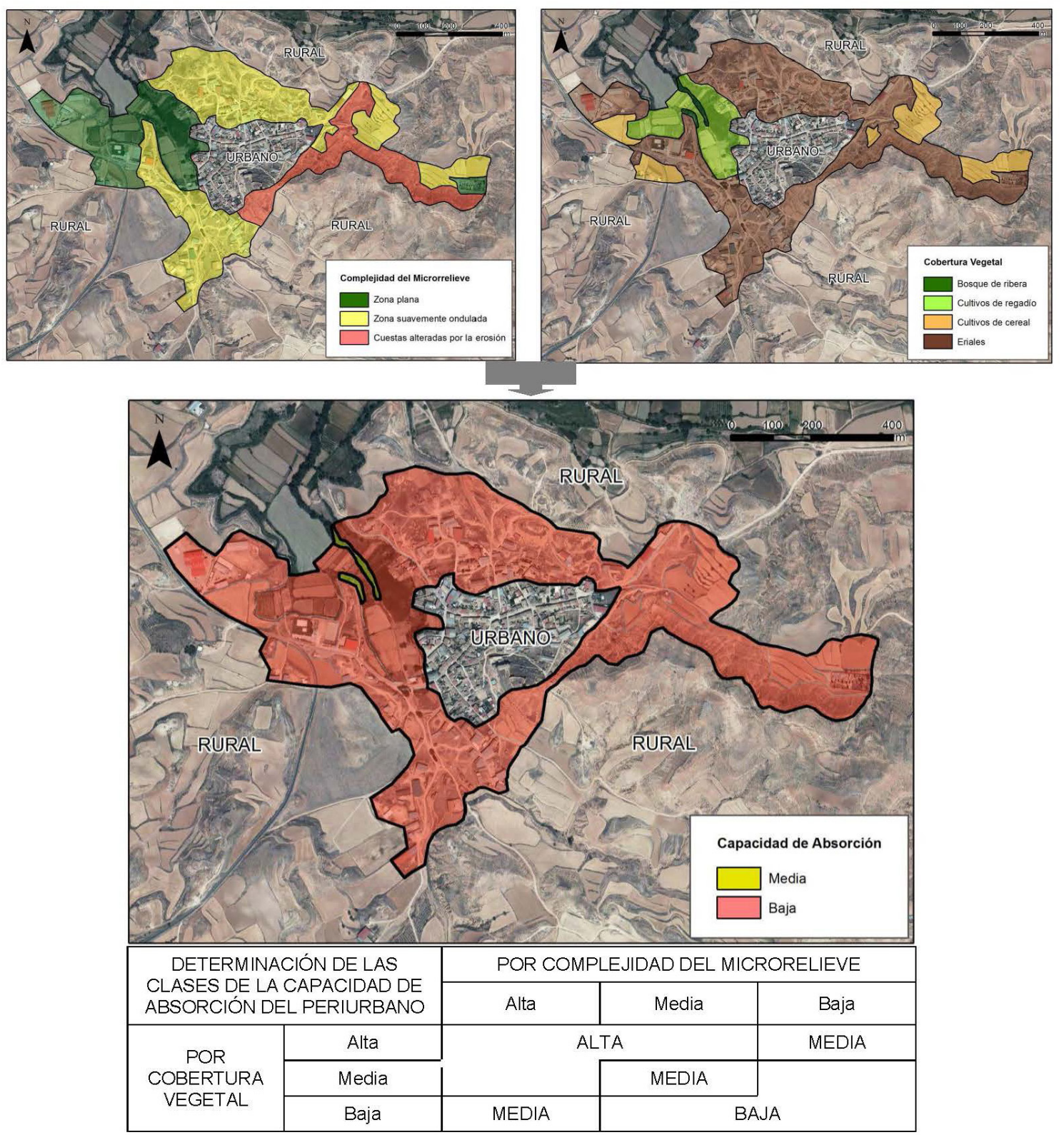

Figura 5. Mapa de capacidad de absorción del periurbano, obtenida por superposición de las capas cartográficas de microrelieve, arriba a la izquierda, y de cobertura vegetal, arriba a la derecha, y aplicando los cruces de la tabla. Fuente: Elaboración propia. 
Fragilidad visual del periurbano. Susceptibilidad al deterioro visual ante actuaciones humanas. Se obtiene superponiendo las capas cartográficas correspondientes a la exposición visual y a la capacidad de absorción; se representa en una nueva capa de clases o recintos de fragilidad visual del periurbano, de acuerdo con los cruces de la figura 6, que responden a esta lógica: a mayor exposición visual y menor capacidad de absorción, mayor fragilidad visual; y viceversa.

Fragilidad paisajística del periurbano. Susceptibilidad al deterioro de los elementos y carácter de las UBP ante actuaciones humanas. Depende, por tanto, de la fragilidad visual y del valor de dichas unidades, y se cartografía superponiendo las capas cartográficas correspondientes a ambos conceptos; las clases de fragilidad paisajística se asignan según los cruces que muestra la figura 6 , que responden a esta lógica: a mayor valor de las UBP y mayor fragilidad visual, correspon- derá mayor fragilidad paisajística; y viceversa. Así se obtendrá una nueva capa cartográfica con los recintos de fragilidad paisajística del periurbano.

Potencial de visualización. Evalúa la capacidad "receptora" de vistas o "potencial de ver" desde un punto, itinerario o área. Se identifica y evalúa a partir de la exposición visual, en función de tres aspectos: profundidad, amplitud y calidad del campo de visión; solo se consideran los lugares con potencial alto.

Fondos escénicos. Barreras visuales permanentes que cierran el horizonte lejano; se identifican los significativos y se cartografían las visuales hacia ellos.

Fragilidad paisajística del borde urbano. La susceptibilidad al deterioro de los tramos del borde, dependerá de su exposición visual y de su valor, pues no aplica la capacidad de absor-

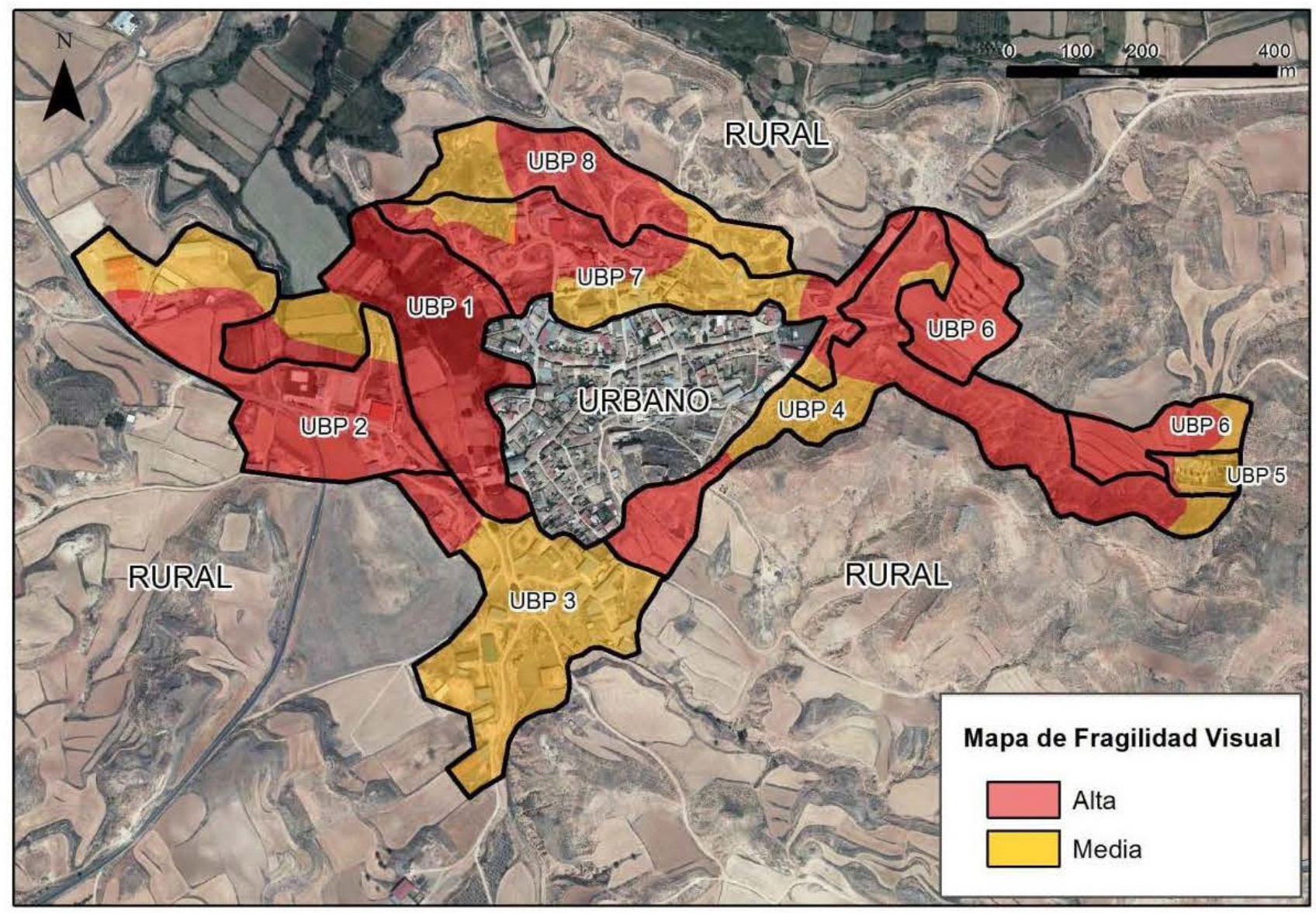

\begin{tabular}{|c|c|c|c|c|}
\hline \multicolumn{2}{|c|}{$\begin{array}{l}\text { DETERMINACIÓN DE LAS CLASES DE } \\
\text { FRAGILIDAD VISUAL DEL PERIURBANO }\end{array}$} & \multicolumn{3}{|c|}{ CLASES DE EXPOSICIÓN VISUAL } \\
\hline & & Alta & Media & Baja \\
\hline \multirow{3}{*}{$\begin{array}{l}\text { CLASES DE } \\
\text { CAPACIDAD DE } \\
\text { ABSORCIÓN }\end{array}$} & Baja & \multirow[t]{2}{*}{ ALTA } & & MEDIA \\
\hline & Media & & MEDIA & \\
\hline & Alta & MEDIA & \multicolumn{2}{|c|}{ BAJA } \\
\hline
\end{tabular}

Figura 6. Mapa de fragilidad visual del periurbano obtenido por superposición de las capas cartográficas correspondientes a la exposición visual y a la capacidad de absorción visual, aplicando los cruces de la tabla. Fuente: Elaboración propia. 
ción. Por ello se obtiene superponiendo las capas cartográficas correspondientes a ambos conceptos, asignado las clases de fragilidad según los cruces de la tabla de la figura 8 , que siguen la lógica apuntada.

Perfiles urbanos. Se identifican los considerados significativos, a partir de la cuenca visual de los accesos y de los lugares de percepción externos al urbano.

Fachadas urbanas: carácter y calidad. El término fachada se aplica en sentido figurado a "lo primero que se ve" cuando se llega a un pueblo o ciudad. Se trata, por tanto, de un concepto asociado a la mayor exposición visual del borde y del perfil urbanos y reflejan el propio perfil, el plano vertical, el ritmo de la trama que conforman las edificaciones y la textura de la escena percibida; en suma las fachadas serían los planos más significativos que se percibe al acceder a la ciudad.

\section{APLICACIÓN AL CASO DE ESTUDIO}

La figura 6 presenta la fragilidad visual del periurbano, obtenida superponiendo las capas cartográficas correspondientes a la exposición visual y a la capacidad de absorción visual; y aplicando los cruces que muestra la tabla.

La figura 7 contiene el mapa de fragilidad paisajística del periurbano, obtenida superponiendo las capas cartográficas correspondientes a la fragilidad visual y al valor de las unidades de la base paisajística; y aplicando los cruces de la tabla.

La figura 4 contiene los puntos con alto potencial de vistas, identificados a partir de la exposición visual, en función de la profundidad, amplitud y calidad del panorama percibido. La misma figura 4 incluye la dirección de las visuales hacia el único fondo escénico significativo antes señalado.

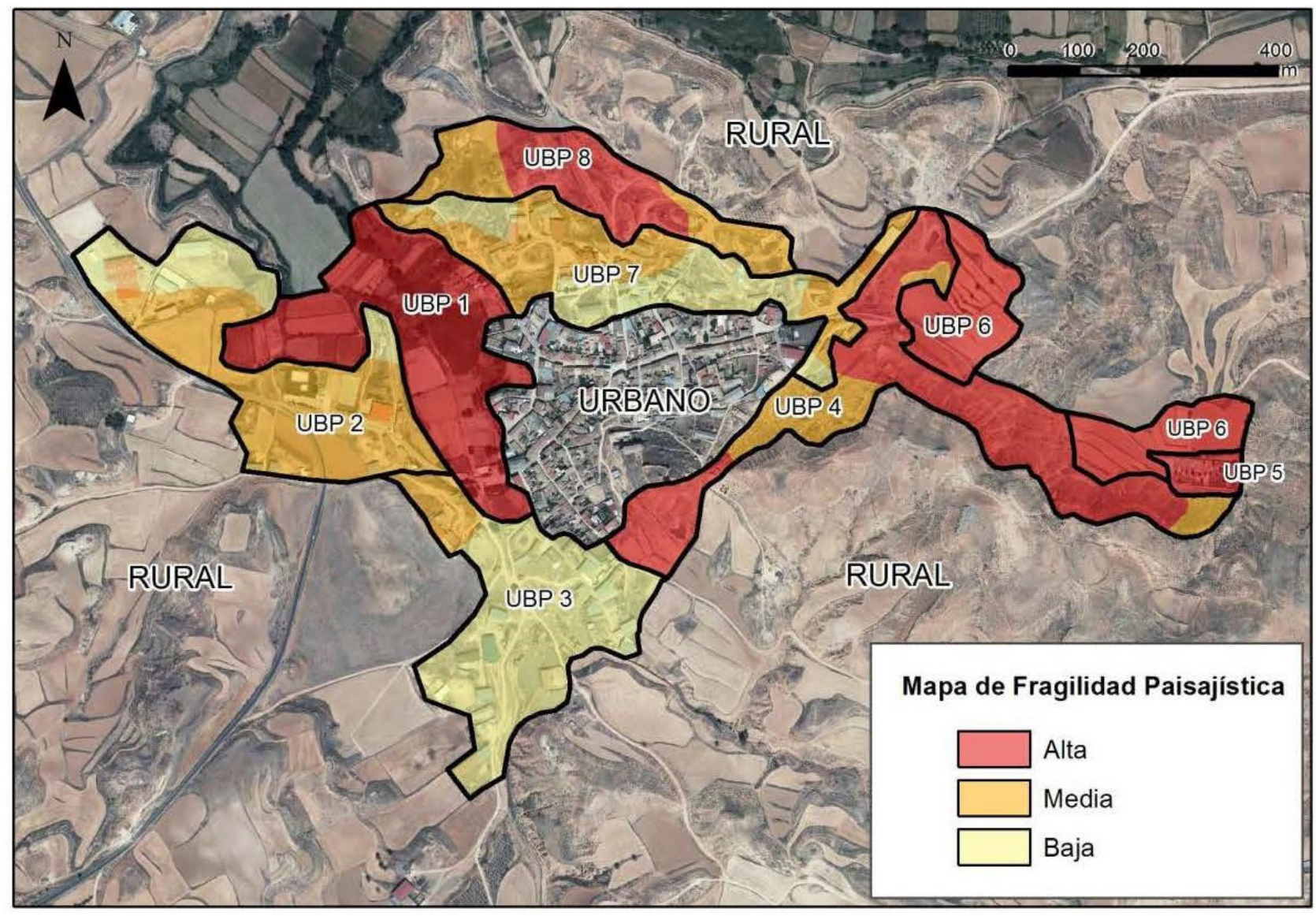

\begin{tabular}{|c|c|c|c|c|}
\hline \multirow{2}{*}{$\begin{array}{c}\text { DETERMINACIÓN DE LAS CLASES DE } \\
\text { FRAGILIDAD PAISAJISTICA DEL } \\
\text { PERIURBANO }\end{array}$} & \multicolumn{3}{|c|}{ VALOR DE LAS UNIDADES DE LA BASE } \\
\cline { 3 - 5 } & AlSAJISTICA \\
\hline \multirow{3}{*}{$\begin{array}{c}\text { CLASES DE FRAGILIDAD } \\
\text { VISUAL }\end{array}$} & Alta & ALTA & Medio & Bajo \\
\cline { 2 - 5 } & Baja & MEDIA & BAJA & \\
\cline { 2 - 4 } & No visible & NULA & NULA & NULA \\
\hline
\end{tabular}

Figura 7. Mapa de fragilidad paisajística del periurbano obtenido por superposición de las capas cartográficas correspondientes a la fragilidad visual y al valor de las unidades de la base paisajística, aplicando los cruces de la tabla. Fuente: Elaboración propia. 
La figura 8 muestra el mapa de fragilidad paisajística de los tramos del borde obtenido por superposición de las capas cartográficas correspondientes a su exposición visual y a su valor, aplicando los cruces que muestra la tabla.

La figura 3 presenta, en rojo, arriba, el perfil urbano más característico del pueblo; y abajo un perfil de la forma banal de su reciente evolución. En negro, el perfil natural del terreno. La figura 9 incluye tres ejemplos representativos de fachadas urbanas, su carácter, calidad y criterios paisajísticos que informarán las propuestas. Obsérvese cómo las fachadas reflejan el perfil urbano, el plano vertical, el ritmo de la trama que conforman las edificaciones y la textura.

\subsection{Dinámica retrospectiva, proyectiva $y$ prospectiva: aspectos históricos, culturales, identitarios}

Retrospectivamente, la situación actual es fruto de la interacción entre el paisaje y sus "formadores". Esta idea queda re- flejada en el modelo por la tira vertical a la izquierda del diagrama (figura 1): aspectos históricos, culturales, identitarios, sin los cuales no se puede entender el paisaje actual y mucho menos proponer medidas de futuro (41).

La evolución futura puede ser proyectiva, basada en las tendencias si no se interviene intencionadamente sobre el paisaje, para cuya determinación se sugiere aplicar el modelo PER (42); o prospectiva, basada en definir la técnica de los escenarios que consiste (24) en identificar estados futuros del paisaje estableciendo hipótesis de cambios no tendenciales en las variables formadoras y las medidas para avanzar hacia ellos.

\section{APLICACIÓN AL CASO DE ESTUDIO}

El paisaje actual del municipio y de la comarca en que se inscribe (Calatayud, Zaragoza), es fruto de la interacción coherente hombre-naturaleza en un largo proceso histórico de ocupación de las vegas por cultivos intensivos de regadío, de sus entornos áridos por cultivos de secano y de las sierras

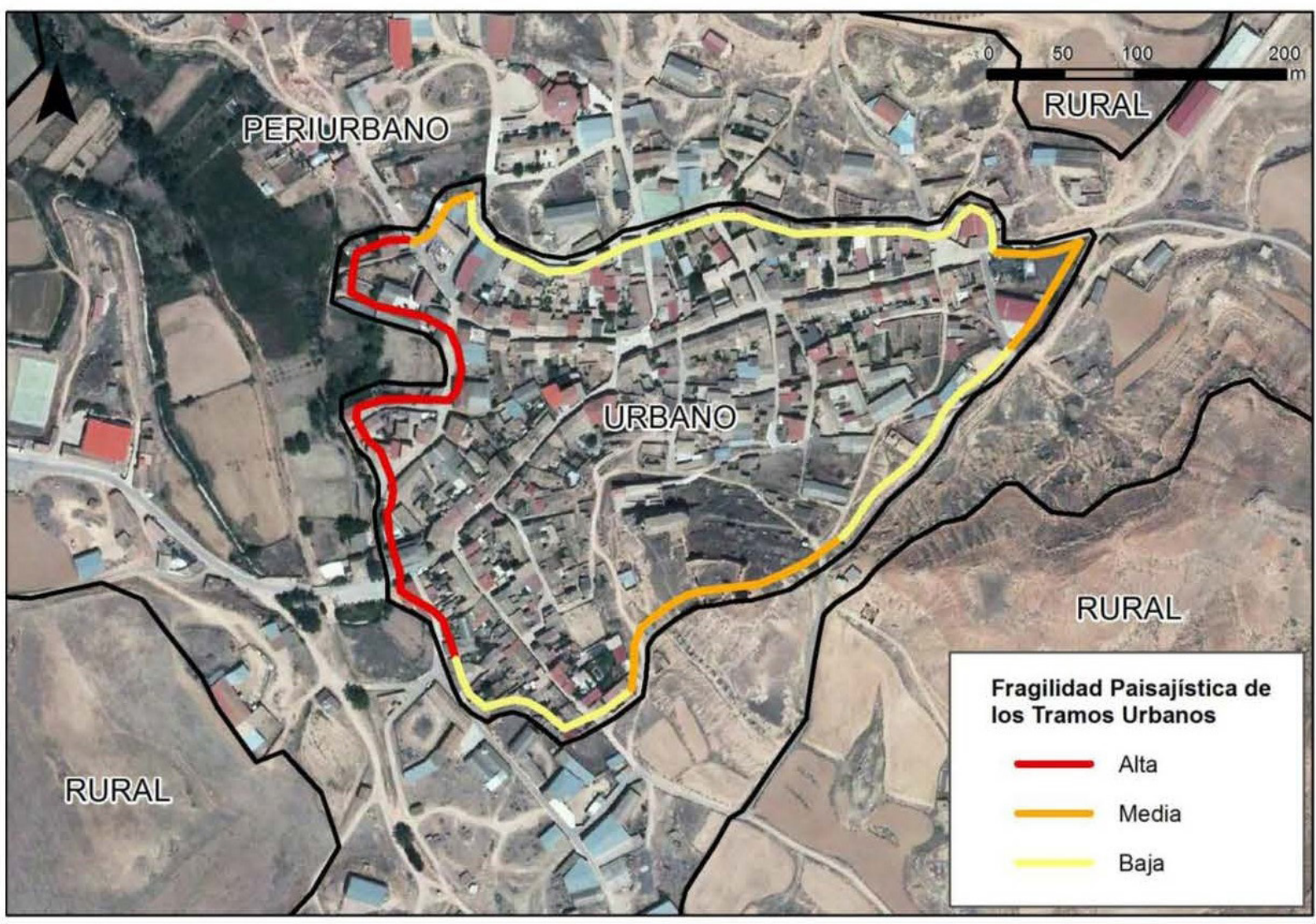

\begin{tabular}{|c|c|c|c|c|}
\hline \multirow{2}{*}{$\begin{array}{c}\text { DETERMINACIÓN DE LAS CLASES DE } \\
\text { FRAGILIDAD PAISAJISTICA DEL BORDE } \\
\text { URBANO }\end{array}$} & \multicolumn{3}{|c|}{ VALOR DE LOS TRAMOS DE PAISAJE DE } \\
\cline { 3 - 5 } & & Alto & Medio & Bajo \\
\cline { 3 - 5 } $\begin{array}{c}\text { CLASES DE EXPOSICIÓN VISUAL } \\
\begin{array}{c}\text { DEL BORDE URBANO DESDE } \\
\text { ACCESOS Y OTROS LUGARES } \\
\text { EXTERNOS AL URBANO }\end{array}\end{array}$ & Alta & ALTA & MEDIA & \\
\cline { 2 - 4 } & No visible & NULA & NULA & \multirow{2}{*}{ NULA } \\
\hline
\end{tabular}

Figura 8. Mapa de fragilidad paisajística de los tramos de paisaje del borde urbano, obtenido por superposición de las capas cartográficas correspondientes a su exposición visual y a su valor, y aplicando los cruces de la tabla. Fuente: Elaboración propia. 
por silvicultura y naturaleza; la ganadería solapada en todos ellos. El resultado es un paisaje armónico y funcional.

Los pueblos suelen ubicarse aledaños a las vegas; su antigüedad e importancia, se explica porque la comarca está en la encrucijada de dos atávicos ejes de comunicación: uno conecta el centro con el noreste peninsular español a través del curso del Jalón; otro conecta el levante con el norte peninsular, a través del Jiloca. La localización, estructura y edificios notables de los pueblos manifiestan los avatares históricos y las culturas asociadas a ellos: iberos, romanos, visigodos, judíos, musulmanes, cristianos, moriscos, mudéjares...; y también su carácter defensivo, frontera con el mundo musulmán en la alta edad media, y entre los poderosos reinos de Aragón y Castilla tras la conquista cristiana. Por ello, destacan los elementos defensivos: murallas y castillos, y los religiosos y culturales: iglesias, torres mudéjares, ermitas, peirones, etc.

La evolución proyectiva del pueblo y su comarca, está marcada por el declive de la agricultura y ganadería tradicionales (y de los elementos histórico culturales), y su sustitución por otras mecanizadas que expulsan población y determinan un gran cambio cultural y paisajístico.

La evolución prospectiva permite imaginar un renacer de los pueblos gracias a las oportunidades de trabajo a distancia y deslocalización de actividades que ofrecen las Tecnologías de la Información y Comunicación (TIC`s) a trabajadores de ciertos sectores; y a una nueva cultura del territorio.

\section{NIVEL PROPOSITIVO DEL MODELO}

Este nivel presenta los elementos conceptuales y metodológicos a través de los cuales se plantea la construcción del paisaje hacia el futuro; el modelo los concreta en los siguientes:
Catálogo de paisaje. Lista ordenada, ilustrada y descrita de los diferentes paisajes existentes en el periurbano, en términos de unidades de paisaje, tramos de borde, fachadas y perfiles urbanos.

Principios, criterios y objetivos. Los principios y criterios relativos al paisaje que se vaya construyendo se orientan en varias direcciones complementarias: conservar los rasgos, el carácter y los valores actuales que lo merezcan; corregir las tendencias indeseables de cambio; recuperar las degradaciones existentes; crear nuevos paisajes coherentes con la evolución social; poner en valor los recursos paisajísticos; e implicar a ciudadanos y agentes socioeconómicos en ello. Los objetivos, enmarcados en los principios y criterios señalados, se vinculan a los problemas y a las potencialidades paisajísticas, y se identifican mediante participación y concertación con los implicados.

Propuestas para el paisaje: en esta tarea se definen las medidas genéricas (de regulación, de intervención y de gestión) para avanzar hacia los objetivos; y también la forma en que se insertan en la planificación urbanística o rural, en los proyectos de ingeniería y arquitectura y en los instrumentos de gestión ambiental.

Unidades de ordenación. Son recintos territoriales para los que se establecen objetivos concretos de calidad paisajística y las propuestas específicas para alcanzarlos; el planificador adoptará los que considere oportuno en cada caso, a partir de las unidades de catálogo, de los recintos de fragilidad paisajística o visual, etc.

Áreas de especial interés paisajístico. Son aquellas que requieren una especial atención por sus valores relevantes, por la forma en que evolucionan o por su estado de degradación.

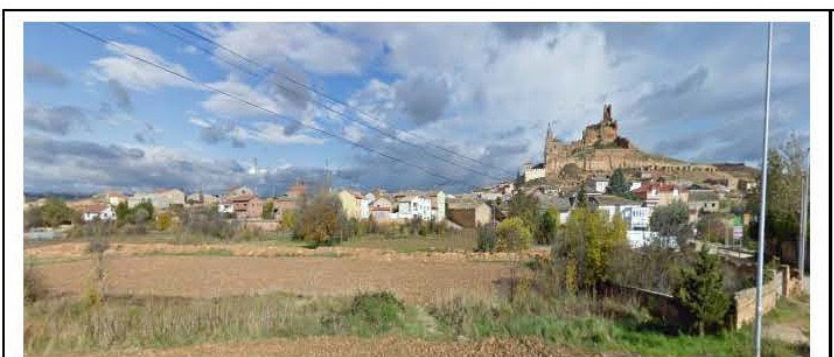

Fachada desde el acceso principal oeste al pueblo, la más representativa; alta calidad.

Criterio: CONSERVAR, previendo intrusiones visuales por volumen, diseño, materiales o color de las nuevas edificaciones o rehabilitación de las existentes.

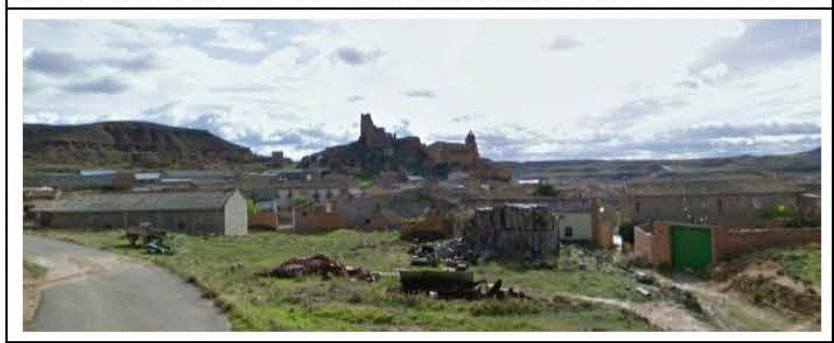

Fachada desde el camino de la vega del río, muy

frecuentado. Calidad media, con degradaciones, pero con elementos históricos identitarios.

Criterio: RECUPERAR el efecto visual negativo producido por el material y color de los tejados de las naves, así como por la deposición de residuos.

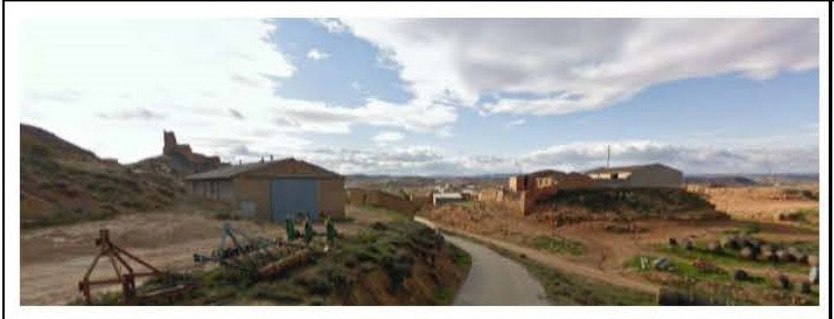

Fachada desde el camino del cementerio, muy frecuentado. Calidad baja, con elementos identitarios poco visibles y síntomas del declive del pueblo.

Criterio: PONER EN VALOR rehabilitando para otros usos las edificaciones deterioradas por el declive poblacional del pueblo y de sus tradiciones

Figura 9. Fachadas urbanas desde lugares accesos y caminos frecuentados, incluyendo criterios paisajísticos. Fuente: Elaboración propia. 
Para éstas se definen, como antes, objetivos de calidad, y propuestas para alcanzarlos.

\section{APLICACIÓN AL CASO DE ESTUDIO}

El catálogo es simplemente la lista ordenada, ilustrada y descrita de las unidades de paisaje, de los tramos de borde, de las fachadas y de los perfiles urbanos.

Los criterios (en los que se inscriben los objetivos y propuestas) añaden, a los expuestos en la figura 9, los que siguen:

- Conservar las UBP 1, 5 y 6 y el tramo Cerro del Castillo.

- Corregir las tendencias evolutivas de las 2, 3 y 7, y del tramo Informal-heterogéneo.

- Recuperar las degradaciones del tramo informal/heterogéneo.

- Crear nuevos paisajes en la zona de expansión de naves agrícolas y logísticas.

- Poner en valor la franja de cuevas de la unidad 4 y los tradicionales pajares de la unidad 8.

- Sensibilizar e implicar a ciudadanía y agentes sobre la importancia y gestión del paisaje.

Como Unidades de Ordenación se proponen las UBP 1, 4, 5 y 6; y como Áreas de Especial Interés Paisajístico, las UBP 2, 3,7 y 8 (figura 2).

\section{DICUSIÓN Y CONCLUSIONES}

La estructura conceptual y metodológica, sencillez y versatilidad del modelo, facilita la comprensión a cualquier interesado del proceso y de los resultados que proporciona, y su aplicación al periurbano de cualquier entidad urbana, sea pueblo o ciudad.

Y ha confirmado las hipótesis iniciales de trabajo, que apuntaban la posibilidad de expresar el carácter subjetivo del paisaje en conceptos lógicos fácilmente comprensibles, de traducirlo a criterios suficientemente objetivables para inventariarlo, diagnosticarlo y planificarlo, y de ordenarlos en una metodología operativa de planificación paisajística. Particularmente clara y convincente resulta la determinación de la fragilidad paisajística; y muy útiles los conceptos de borde, fachada y perfil urbanos en la ordenación del periurbano.

Pero la técnica de superposición de capas cartográficas que utiliza y la facilidad de aplicación con una herramienta GIS ha puesto de manifiesto el riesgo de hacer una superposición mecánica generando resultados intermedios y finales incoherentes, alejados de lo que dicta la lógica y el propio punto de vista de los ciudadanos. Para evitarla conviene hacer una aplicación iterativa del modelo, avanzando y retrocediendo sobre lo realizado a la luz del sentido común y de la participación social.

En consecuencia, la mejor aplicación del modelo consiste en entenderlo como método de aprendizaje sobre el paisaje, sobre la forma en que ha evolucionado hasta la situación actual y sobre su construcción a futuro.

El trabajo ha sugerido profundizar en la conceptualización y delimitación del espacio periurbano, así como en la forma de articularlo con las dos referencias que lo delimitan: el urbano y el rural.

\section{REFERENCIAS}

(1) Alexis, S. \& Dumont, J. (2005): Les 7 familles de paysages en Rhône-Alpes. Des paysages pluriels pour un territoire singulier, Direction Régionale de l'Environnement, Rhône-Alpes, Lyon, Francia.

(2) Tveit, M., \& Ode Sang, A. (2014). Landscape assessment in metropolitan areas - developing a visual indicator-based approach. SPOOL, 1(1). doi: 10.7480/spool.2013.1.641.

(3) Tress, G., Tress, B., Harms, B., Smeets, P. \& Van der Valk, A. (Eds.) (2004). Planning Metropolitan Landscapes - Concepts, Demands, Approaches. Wageningen, Netherlands: Alterra.

(4) Van den Brink, A., Van der Valk, A., \& Van Dijk, T. (2007). Planning and the Challenges of the Metropolitan Landscape: Innovation in the Netherlands. International Planning Studies, 11, 147-165.

(5) Capel, H. (1975). La definición de lo urbano. Estudios geográficos, 36(138), 265.

(6) Hildenbrand Scheid, A. (2016). Tesis doctoral: Gobernanza y planificación territorial en las áreas metropolitanas. Análisis comparado de las experiencias recientes en Alemania y de su interés para la práctica en España. Sevilla. Universidad de Sevilla.

(7) Martner, C. (2015). Expansión dispersa, ciudad difusa y transporte: El caso de Querétaro, México. Revista EURE-Revista de Estudios Urbano Regionales, 42(125).

(8) Gražulevičiūtė-Vileniškè, I., Zaleskienè, E. (2014). Landscape Research Trends and Some Insights from Rurban Landscape. Environmental Research, Engineering and Management. Vol 67, $n^{\circ} 1$.

(9) Council of Europe. (2000). European landscape convention. In Report and Convention. Council of Europe, 200o, Zoido, 2004.

(10) Naranjo, F. Z. (2001). La Convención Europea del Paisaje y su aplicación en España. Ciudad y territorio: Estudios territoriales (128), 275-282.

(11) UNESCO y Federación Internacional de Arquitectos Paisajistas. (2012). Iniciativa Latinoamericana del Paisaje. Medellín (Colombia).

(12) De Pisón, M., \& Herráiz, S. (2000). Estudios sobre el paisaje. UAM Ediciones; Fundación Duques de Soria.

(13) Santos y Ganges, L. (2002). Las nociones de paisaje y sus implicaciones en la ordenación. Ciudades, 7 (2002-2003), pp. 41 a 68.

(14) Ministère de l'Écologie, du Développement Durable et de l’Énergie. (2015). Les Atlas de paysages. París.

(15) Olmo, R. M., \& i Coscuela, A. T. (2006). El paisaje y la gestión del territorio: criterios paisajísticos en la ordenación del territorio y el urbanismo. Diputació Provincial de Barcelona.

(16) Domínguez, J. M. E., \& Guerton, J. B. (2008). Plan General de Ordenación Urbana de Segovia, 2007. Urban, (13), 68-86. 
(17) Laboratorio de Planificación Territorial, Universidad Católica de Temuco (2010): Plan Regulador Intercomunal Gran Temuco, IX Región, Ministerio de Vivienda y Urbanismo, Temuco, Chile.

(18) Espinosa, E. M. M. (2013). Acondicionamiento paisajístico de los accesos a pequeñas poblaciones. Ensayo metodológico en el valle de Lecrín (Granada). Ería, (92).

(19) Constant, C. (2012). The Modern Architectural Landscape. Ed. University of Minnesota Press.

(20) Spirin, A. (1998): The Language of Landscape, Yale University Press, New Haven and London, UK.

(21) Theano, S. (2001). Towards a theory of the landscape: the Aegean landscape as a cultural image. Landscape and urban planning, volumen: $57 \mathrm{n}^{0} 3$ pp. 197-208, Elsevier, Amsterdam.

(22) Serrano Giné, D. (2015), "Valoración escénica de paisaje periurbano con utilidad en planeamiento territorial. Estudio de caso en la Región Metropolitana de Barcelona”, Investigaciones Geográficas, Boletín, núm. 88, Instituto de Geografía, UNAM, México, pp. 109-121.

(23) Antrop, M., \& Van Eetvelde, V. (2000). Holistic aspects of suburban landscapes: visual image interpretation and landscape metrics. Landscape and urban planning, 5o(1), 43-58, doi: http://dx.doi.org/10.1016/So169-2046(00)ooo79-7.

(24) Gómez Orea, D. y Gómez Villarino, A. (2013). Ordenación territorial. Mundi-Prensa. Madrid.

(25) Lewin, K., \& Gold, M. E. (1946). The complete social scientist. American Psychological Association.

(26) Mata Olmo, R. y Sanz Herráiz, C. (dirs.) (2003): Atlas de los Paisajes de España. Madrid, Ministerio de Medio Ambiente.

(27) Gobierno de Aragón. (2003). Comarca de Comunidad de Calatayud. Colección Territorio 8. Departamento de Presidencia y Relaciones Institucionales. Gobierno de Aragón. Zaragoza.

(28) Gobierno de Aragón. (2009). Mapa de paisaje de las Comarcas de Aragón. Departamento de Política Territorial, Justicia e Interior. Gobierno de Aragón.

(29) Instituto Geográfico Nacional (IGN). (2012). Ortofotografías aéreas del vuelo del año 2012, de la red PNOA (Plan Nacional de Ortofotografía Aérea). Instituto Geográfico Nacional. Madrid.

(30) Ministerio de Medio Ambiente. 2004. Mapa Forestal de España (MFE50), 1:50.ooo. Provincia de Zaragoza. Madrid.

(31) Ministerio de Medio Ambiente. 2004. Mapa de Cultivos y de Aprovechamientos, escala 1:50.000. (2008). Ministerio de Agricultura del Gobierno de España. Madrid.

(32) Peña, J. L., \& Lozano, M. V. (2004). Las unidades del relieve aragonés. Geografía Física de Aragón, Aspectos Generales Y Temáticos, 3-14.

(33) Blasco Font de Rubinat, J. (2000). Los artefactos y sus proyectos. Edicions UPC.

(34) Ode, Å., Tveit, M., \& Fry, G. (2008). Capturing Landscape Visual Character Using Indicators: Touching Base with Landscape Aesthetic Theory. Landscape Research, 33(1), 89-118.

(35) Ode, Å., Hagerhall, C. M., \& Sang, N. (2010). Analysing Visual Landscape Complexity: Theory and Application. Landscape Research, 35(1), 111-131.

(36) Banks, M., \& Zeitlyn, D. (2015). Visual methods in social research. Sage.

(37) Spirin, A. W. (1998). The Language of Landscape. New Haven, CT: Yale University Press.

(38) Otero, I., Casermeiro, M., Ezquerra, A., Esparcia, P. (2007). Landscape evaluation: Comparision of evaluation methods in a region of Spain. Journal of Environmental Management (n. ${ }^{\circ} 85$ ), pp. 204-214.

(39) Arriaza, M., Cañas Ortega, J.F., Cañas Madueño, J.A., Ruiz Avilés, P. Assessing the visual quality of rural landscapes Landscape and Urban Planning (n. ${ }^{\circ}$ 69) (2004), pp. 15-125.

(40) Cañas, I., Ayuga, E., Ayuga, F. (2009). A contribution to the assessment of scenic quality of landscapes based on preferences expressed by the public. Land Use Policy 26 (4), 1173-1181.

(41) Gómez Villarino, M. y Gómez Villarino, T. (2016). Propuesta metodológica para estudiar el paisaje urbano. Aplicación de caso en Temuco, Chile. Ciudad y Territorio. $\mathrm{n}^{0}$ 190. pp. 675-695.

(42) OCDE. (1994). OCDE Core Set of Environmental Indicators, Paris. 